\title{
Descrição de larva de Zenithoptera anceps (Odonata, Libellulidae)
}

\author{
Janira Martins Costa ${ }^{1}$, José Pujol-Luz ${ }^{2} \&$ Luci L. P. B. Regis ${ }^{3}$
}

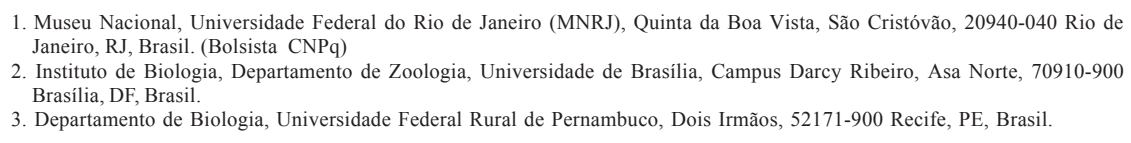

ABSTRACT. Description of the larva of Zenithoptera anceps (Odonata, Libellulidae). The larva of Zenithoptera anceps Pujol-Luz, 1993 is described and figured for the first time. A comparison among the known larvae of Palpopleurinae is presented. A key to the larvae of the neotropical genera of Palpopleurinae is added.

KEYWORDS. Libellulidae, Palpopleurinae, Zenithoptera, taxonomy, Neotropical.

\section{INTRODUÇÃO}

Dentre os Palpopleurinae neotropicais, somente Diastatops Rambur, 1842, com nove espécies e Perithemis Hagen, 1861, com 12, têm suas larvas conhecidas: Diastatops intensa Montgomery, 1940 (CosTA et al.,1999), D. obscura (Fabricius, 1775) (SANTos et al., 1993), Perithemis domitia (Drury, 1773) (NeEdham et al., 2000), P. electra Ris, 1930 (SAntos, 1970), P. icteroptera (Selys, 1857) (Von Ellenrieder \& MuZón, 1999), P. mooma, Kirby, 1889 (SAntos, 1973), P. rubita Dunkle, 1982 (DunkLE, 1982) e P. thais Kirby, 1889 (Spindola et al., 2001).

O gênero Zenithoptera Bates, 1869 compreende quatro espécies válidas: $Z$. americana (Linnaeus, 1758), $Z$. anceps Pujol-Luz, 1993, Z. lanei Santos, 1941 e Z. viola Ris, 1910, sendo $Z$. anceps endêmica do Brasil, ocorrente na mata Atlântica e na Amazônia brasileira (PuJoL-Luz, 1997).

$\mathrm{O}$ conhecimento da larva de $Z$. anceps, descrita a seguir, é importante para a compreensão das relações entre os gêneros pertencentes a Palpopleurinae.

\section{MATERIAL E MÉTODOS}

As larvas de Zenithoptera anceps foram capturadas em um pequeno "olho d'água" na localidade de Dois Irmãos ( $08^{\circ} 01^{\prime} 00^{\prime}$ ” $\mathrm{S}$; $\left.34^{\circ} 56^{\prime} 00^{\text {” }} \mathrm{W}\right)$, município de Recife, Pernambuco, Brasil. Foram capturados 10 indivíduos machos e uma fêmea de último instar utilizando-se peneiras de malha fina, em seguida separados e mantidos individualmente em caixa de isopor telada, para evitar o canibalismo. No laboratório, foram alimentados com larvas de Aedes (Stegomyia) aegypti (Linnaeus, 1762) e Culex quinquefasciatus Say, 1823. Após 48h da captura, somente a fêmea de último instar emergiu. Esta foi mantida viva até sua morte natural, o que ocorreu três dias após sua captura.

Foram capturadas no mesmo habitat outras larvas de Odonata: Diastatops intensa, Erythrodiplax fusca (Rambur, 1842), Pantala flavescens (Fabricius, 1798) e Planiplax phoenicura Ris, 1912.

A análise do solo (substrato) foi realizada no Laboratório de Fertilidade do Solo, Departamento de Agronomia da UFRPE e a da água no Laboratório da
Secretaria de Recursos Hídricos do Estado de Pernambuco.

A fórmula mandibular foi determinada de acordo com Watson (1956). O material está depositado no Museu Nacional, Rio de Janeiro (MNRJ) e na Universidade Federal Rural de Pernambuco (UFRPE).

\section{Zenithoptera anceps Pujol-Luz, 1993 (Figs. 1-11)}

Diagnose. A larva de Zenithoptera anceps é característica de Libellulidae (fig.1), muito similar a das outras espécies de Palpopleurinae pela presença de espinhos laterais nos segmentos abdominais VIII-IX (fig.1), porém difere pelo tamanho dos mesmos no segmento VIII, desenvolvido em $Z$. anceps, e ausência de espinho no dorso do segmento IX (figs.1, 7).

Coloração da larva de último instar: castanho-claro.

Cabeça (figs.1,2) rombóide, ligeiramente mais larga que o tórax em vista dorsal; região pós-ocular levemente arredondada com pequenas setas espiniformes e um conjunto de outras ligeiramente maiores, dispostas dorsalmente entre os olhos; olhos pequenos, semicirculares; antenas com sete antenômeros (fig. 3), primeiro e segundo de igual tamanho, terceiro, quarto $\mathrm{e}$ quinto também de igual tamanho, sexto o maior, sétimo ligeiramente menor que o sexto. Mandíbula esquerda (fig. 4) com quatro incisivos e três molares; mandíbula direita (figs. 5, 6) com quatro incisivos, três molares e o dente y; fórmula mandibular: L $12340 \mathrm{abd} / \mathrm{R} 1234$ y abd. Lábio mais largo que longo, quando dobrado alcança a metade do comprimento do segundo mesosterno; prémento (fig. 7) com 12-13 setas prementais, dois pequenos espinhos em cada lado da junção com o palpo labial; palpo labial (fig. 8) com sete setas além do espinho móvel; este, pequeno, aproximadamente do mesmo tamanho que as setas.

Tórax (fig.1) com apófises supracoxais arredondadas e 5-7 pequenas setas espiniformes; tecas alares ligeiramente divergentes, alcançando a metade do comprimento do sexto segmento; pernas longas e delgadas, ultrapassando o comprimento do abdômen; fêmures com duas manchas escuras (fig. 1). 

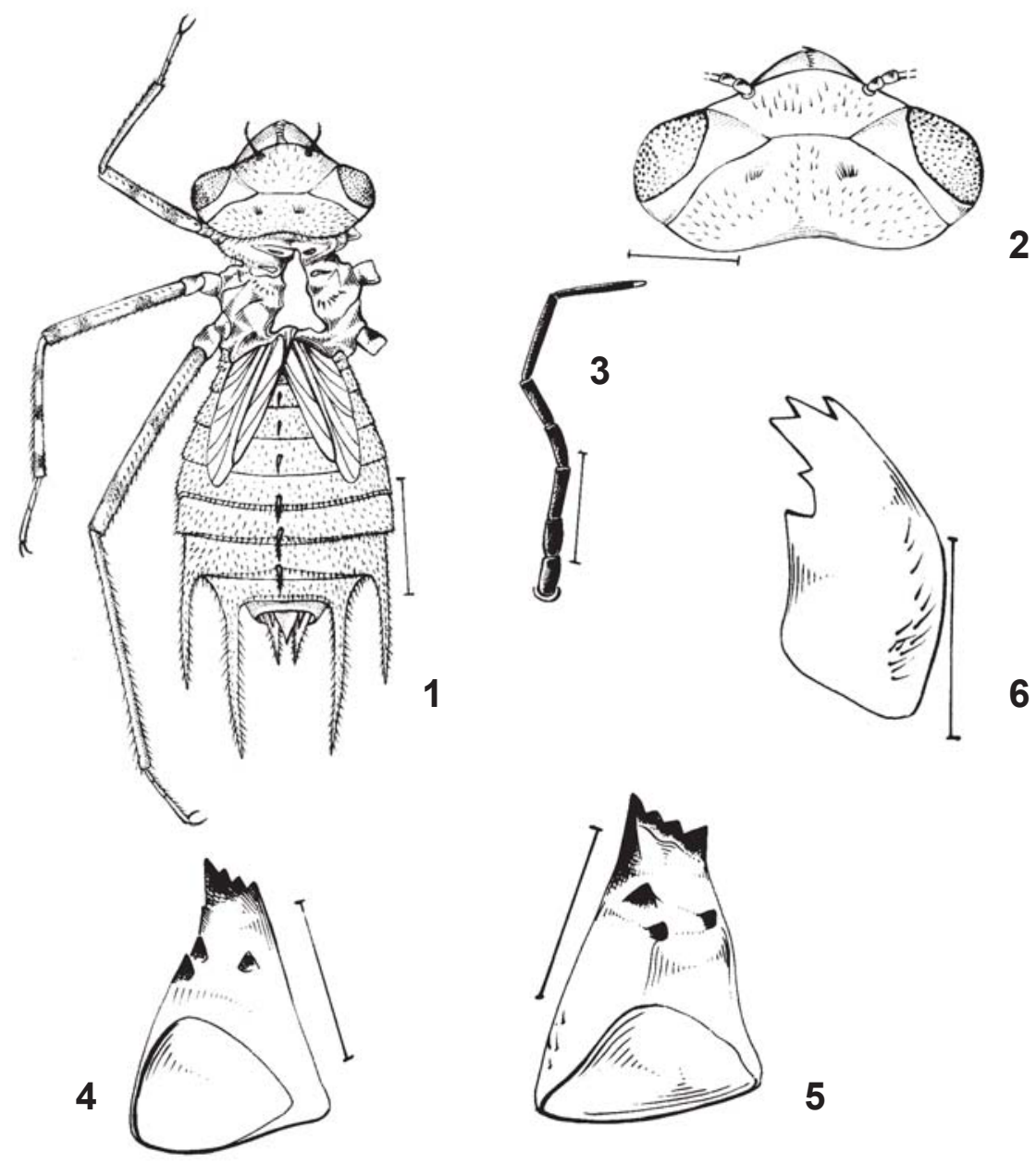

2

Figs. 1-6. Zenithoptera anceps Pujol-Luz, 1993, larva de último instar: 1, aspecto geral; 2, cabeça, vista dorsal; 3, antena; mandíbula: 4, esquerda, vista ventral; 5, direita, ventral; 6, direita, dorso-lateral. Escalas: $2 \mathrm{~mm}$, fig. 1 ; $1 \mathrm{~mm}$, figs. 2, 4-6; 0,5 mm, fig. 3 .

Abdômen (figs. 1, 9, 11) cilíndrico; segmentos VVIII aproximadamente da mesma largura; margem dorsolateral provida de pequenas cerdas concentradas em toda sua extensão; espinhos dorsais presentes do terceiro ao oitavo segmento (figs. 1, 9,11), os três primeiros curtos, em série crescente de tamanho, os do quinto segmento ligeiramente menores que os do sexto e dirigidos para cima, do sexto ao oitavo curvos, aproximadamente do mesmo tamanho e dirigidos para trás (figs. 9, 11); espinhos laterais nos segmentos VII-IX (figs. 1, 9), os do sétimo segmento reduzidos, do oitavo e nono desenvolvidos, sendo os do nono ligeiramente maiores que os do anterior. Apêndices anais (fig. 10) cônicos, cercos curtos, metade do comprimento do epiprocto, pontiagudos; epiprocto triangular com extremidade distal espiniforme; paraproctos longos, duas vezes o comprimento do epiprocto (figs. 1, 10).

Dimensões, em mm. Comprimento total, 14; cabeça: comprimento 2,60, largura 3,75; olhos: comprimento 1,45, largura 0,65 ; comprimento total das antenas 1,66 ; comprimento de cada antenômero: 0,19/0,17/ 0,26/0,21/ $0,24 / 0,42 / 0,43$; comprimento do pré-mento 2,00; largura máxima do pré-mento 2,20; palpo labial: comprimento 1,90, largura máxima 1,35; comprimento da teca alar: anterior
3,80, posterior 3,50; comprimento dos fêmures: F1 2,30, F2 3,00, F3 4,50; comprimento das tíbias: T1 2,50, T2 2,90, T3 4,80; abdômen: comprimento 6,60, largura máxima 4,40; comprimento do espinho lateral: VIII 3,1, IX 3,3; comprimento do epiprocto 0,1 ; comprimento do paraprocto 0,2 ; comprimento do cerco 0,5 .

O habitat onde as larvas foram coletadas possui substrato arenoso, rico em macrófitas aquáticas e situado aproximadamente a $4 \mathrm{~m}$ do açude do Meio. A análise do solo apresentou os seguintes resultados: pH 5,11 (Ref. água-1:2,5); $\mathrm{P} \quad 1,00\left(\mathrm{mg} \cdot \mathrm{dm}^{-3}\right) ; \mathrm{K}^{+} 0,06 ; \mathrm{Na}^{+} 0,07$; $\mathrm{Ca}^{2+}+\mathrm{Mg}^{2+} 0,70 ; \mathrm{Ca}^{2+} 0,45 ; \mathrm{Al}^{3+} 0,45\left(\mathrm{cmol} \cdot \mathrm{dm}^{-3}\right)$. A análise da água, em dias alternados, apresentou $\mathrm{pH}$ 5,72; condutividade elétrica $42 \mu \mathrm{S} / \mathrm{cm}$; oxigênio dissolvido $0,2 \%$; temperatura da água entre $22^{\circ} \mathrm{C} \mathrm{e} 27^{\circ} \mathrm{C}$; salinidade $23,59 \%$. A temperatura do ar variou entre $27^{\circ} \mathrm{C}$ e $31^{\circ} \mathrm{C}$ e a umidade entre $85 \%$ e $93 \%$.

A vegetação em volta do "olho d'água" apresentase compacta, formando uma cobertura parcial que não permite a penetração direta dos raios solares. As principais famílias de vegetais representativas da área são: Cecropiaceae (Cecropia sp.), Musaceae (Heliconia sp.) e Melastomataceae (Miconia sp. e Clidemia sp.). Adultos de $Z$. anceps foram observados nesse habitat 

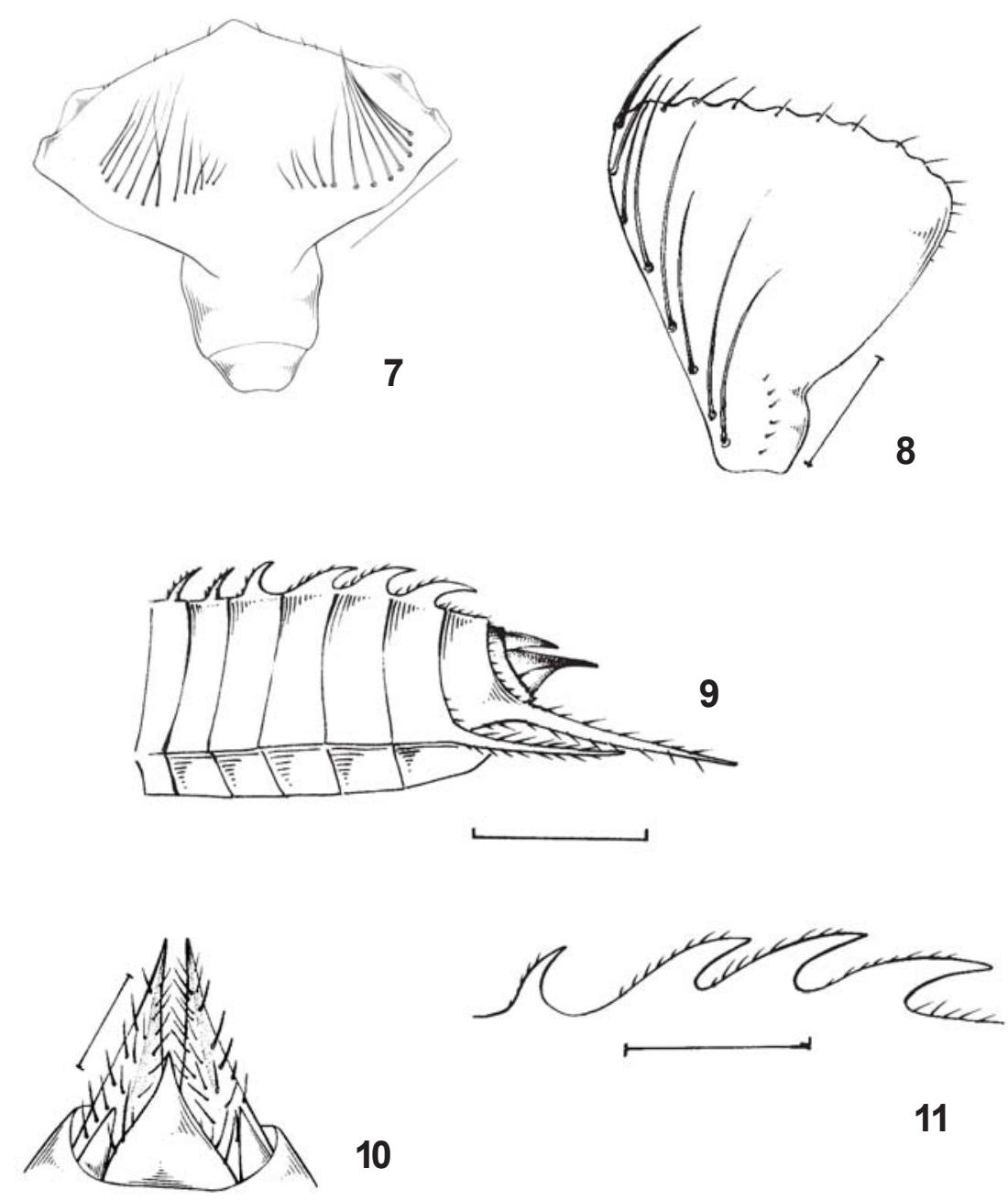

10

Figs. 7-11. Zenithoptera anceps Pujol-Luz, 1993: 7, premento, vista ventral; 8, palpo labial, dorsal; 9, abdômen, lateral; 10, apêndices anais; 11, espinhos dorsais dos segmentos V-VIII. Escalas: $2 \mathrm{~mm}$, fig. 9; $1 \mathrm{~mm}$, figs. 7, 8, 11; 0,5 mm, fig.10.

durante quatro dias. Essa observação corrobora aquela realizada por PujoL-Luz (1998) quanto à territorialidade de machos desta espécie.

Material examinado. BRASIL, Pernambuco: Recife, Reserva Florestal do Açude do Prata, Dois Irmãos, 6-9.II.2001, 11

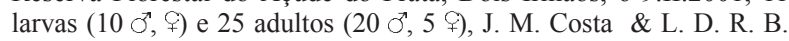
Régis leg. Seis larvas e 15 adultos encontram-se depositados no MNRJ e cinco larvas e dez adultos na UFRPE.

Distribuição geográfica. Brasil (Amazonas, Pará, Rondônia, Maranhão, Pernambuco, Alagoas, Bahia, Espírito Santo, Rio de Janeiro, São Paulo e Paraná).

Chave para identificação dos gêneros das larvas conhecidas de Palpopleurinae neotropicais

1. Espinhos dorsais nos segmentos abdominais III-VIII; espinhos laterais dos segmentos abdominais VIII e IX desenvolvidos, os do IX uma vez e meia o comprimento do VIII; paraproctos duas vezes o comprimento do epiprocto Zenithoptera

Espinhos dorsais nos segmentos abdominais III-IX; espinhos laterais do segmento abdominal IX cerca de quatro vezes o comprimento do VIII; paraproctos do mesmo tamanho ou ligeiramente maiores que o epiprocto ... 2

2. Espinhos laterais do segmento abdominal IX curtos; palpos labiais com digitações rasas; cabeça sem tubérculos; olhos pequenos, semicirculares; epiprocto e paraproctos do mesmo tamanho Perithemis

Espinhos laterais do segmento abdominal IX desenvolvidos, 4 a 5 vezes o comprimento dos espinhos do VIII segmento; palpos labiais com digitações profundas; cabeça com 2 tubérculos; olhos cônicos com extremidades pontiagudas; epiprocto ligeiramente menor que os paraproctos

Diastatops

Agradecimentos. Ao CNPq e FAPERJ, pelos auxílios concedidos. À UFRPE, pelo apoio para as pesquisas de campo. Ao Sr. Luiz Antônio Alves Costa (MNRJ), pelas ilustrações.

\section{REFERÊNCIAS BIBLIOGRÁFICAS}

Costa, J. M.; Souza-Franco, G. M. \& Takeda, A. M. 1999. Descrição da larva de Diastatops intensa Montgomery, 1940 e morfologia dos diferentes estádios de desenvolvimento (Odonata: Libellulidae). Boletim do Museu Nacional, N. Sér., Zoologia, Rio de Janeiro, 410:1-14. 
Dunkle, S. W. 1982. Perithemis rubita spec. nov., a new dragonfly from Ecuador (Anisoptera: Libellulidae). Odonatologica, Utrecht, 11(1):33-39.

Needham, J. G.; Westfall, N. T., JR. \& May, M. L. 2000. Dragonflies of North America, Gainesville, Scientific Publishers. 940p.

Pujol-Luz, J. R. 1997. Variação de coloração das asas e distribuição geográfica do gênero Zenithoptera Bates in Selys (Odonata, Libellulidae). Revista da Universidade Rural, Série Ciência Vida, Rio de Janeiro, 19(1-2):13-26. 1998. Observações sobre o comportamento territorial de machos de Zenithoptera anceps Pujol-Luz, 1993 (Odonata: Libellulidae). Revista da Universidade Rural, Série Ciência Vida, Rio de Janeiro, 20(1-2):97-102.

Santos, N. D. 1970. Contribuição ao conhecimento da fauna do Estado da Guanabara. 76 - Descrição da ninfa de Perithemis electra Ris, 1930 e notas sobre o macho (Odonata: Libellulidae). Atas da Sociedade de Biologia do Rio de Janeiro, Rio de Janeiro, 14(3/4):49,50.

1973. Contribuição ao conhecimento da fauna do Estado da Guanabara e arredores. 84 - Descrição da ninfa de
Perithemis mooma Kirby, 1889 (Odonata: Libellulidae). Atas da Sociedade de Biologia do Rio de Janeiro, Rio de Janeiro, 16(2/3):71, 72 .

Santos, N. D.; Costa, J. M. \& Pujol-Luz, J. R. 1993. Descrição da larva de Diastatops obscura (Fabricius) (Odonata, Libellulidae). Revista Brasileira de Zoologia, Curitiba, 10(3):467-472.

Spindola, L. de A.; Souza, L. O. I. \& Costa, J. M. 2001. Descrição da larva de Perithemis thais Kirby, 1889, com chave para identificação das larvas das espécies conhecidas do gênero citadas para o Brasil (Odonata: Libellulidae). Boletim do Museu Nacional, N. Sér., Zoologia, Rio de Janeiro, 442:1-8.

Von Ellenrieder, N. \& Muzón, J. 1999. The Argentinean species of the genus Perithemis Hagen (Anisoptera: Libellulidae). Odonatologica, Bilthoven, 28(4):385398.

Watson, M. C. 1956. The utilization of mandibular armature in taxonomic studies of anisopterous nymphs. Transactions of the American Entomological Society, Philadelphia, 81:155-209. 